\title{
Adult Burkitt lymphoma: An institutional experience with a uniform chemotherapy protocol
}

\author{
Mukesh Patekar,Ajay Gogia,Akash Tiwari, Lalit Kumar,Atul Sharma, Soumya Ranjan Mallick', Mehar Chand Sharma', \\ Sanjay Thulkar', Ritu Gupta ${ }^{3}$
}

\begin{abstract}
Background: Burkitt lymphoma $(\mathrm{BL})$ is treated with short, intensive, noncross resistant multidrug chemotherapy regimens. The management of this aggressive lymphoma is a challenge in our resource-limited setting, and the published data from India is scarce. Aim: This retrospective study aims to evaluate the clinical features and treatment outcomes in adult patients with BL treated with uniform chemotherapy, cyclophosphamide, vincristine, doxorubicin, methotrexate, ifosfamide, etoposide, cytarabine (CODOX-M/IVAC) protocol ( \pm Rituximab). Materials and Methods:The hospital records between $201 \mathrm{I}$ and $20 \mathrm{I} 7$ were reviewed to identify adult patients (age $\geq 18$ years) who were treated with CODOX-M/IVAC protocol ( \pm Rituximab). The demographic and clinical details, treatment, outcomes, and toxicity were recorded from the patient's prospectively maintained case records. Results: Eighteen patients were included in this study. The median age was 38 years with male:female ratio 3.5:I. The majority of patients were high risk (I4/I8). All patients had extranodal site of involvement. The treatment completion rate was $83.3 \%$. The overall response rate $=77.8 \%$ including complete response rate $=66.7 \%$. Five patients $(27 \%)$ had progressive disease on therapy. The estimated 2 -year overall survival and event-free survival were $73 \%$ and $68.4 \%$, respectively. The most common toxicity was myelosuppression (grade v3/4 neutropenia $=88.8 \%$, grade $3 / 4$ thrombocytopenia $=77.7 \%$, and grade $3 / 4$ anemia $=66.6 \%$ ), febrile neutropenia was seen in $66.6 \%$ cases. Most common nonhematological toxicity was mucositis (grd3/4 = 33.3\%). No toxic death was seen. Conclusion:This one of the first retrospective analyses of treatment outcomes from India suggests that our patients are demographically and clinically similar to the western counterpart. The treatment completion rate is high despite significant toxicity. BL has a good outcome if treated adequately.

Key words: Burkitt lymphoma, cyclophosphamide, cytarabine/uniform protocol/treatment, doxorubicin, etoposide, ifosfamide, methotrexate, outcome, vincristine
\end{abstract}

\section{Introduction}

Burkitt lymphoma (BL) is a highly aggressive B-cell NonHodgkin lymphoma (NHL). It was the first tumor which was discovered to be associated with a viral infection (Epstein-Barr virus) and a chromosomal translocation which activates an oncogene (c-MYC). The BL and Burkitt leukemia is considered to be the part of the same disease spectrum. ${ }^{[1]}$ WHO describes three distinct clinical forms of BL: endemic (African), sporadic (nonendemic), and immunodeficiency-associated. ${ }^{[1]} \mathrm{BL}$ is predominantly a disease of childhood, but it is also seen in the adult population. The diagnostic and therapeutic principles of BL in the adult are an extension of those used in the pediatric population.

The challenges of diagnosis and treatment of an aggressive lymphoma, like BL, is unique in low-income and resource-limited settings. The paucity of an adequate molecular diagnostic facility, trained hematopathologist, and limited access to health facility is an impediment in the adequate care of these patients. The data of the outcome of these patients from the low-income countries are sparse. Therefore, we, hereby present our experience of treating patients with $\mathrm{BL}$ with a uniform chemotherapy protocol from a tertiary care center in North India.

\section{Materials and Methods}

\section{Patient information}

We have retrospectively reviewed the case records of patients with BL registered in our clinics. The database included search from the digital records and case files of patients with a diagnosis of BL. We identified 22 cases of adult BL (age $\geq 18$ years) during 2011-2017. Eighteen patients received cyclophosphamide, vincristine, doxorubicin, methotrexate, ifosfamide, etoposide,

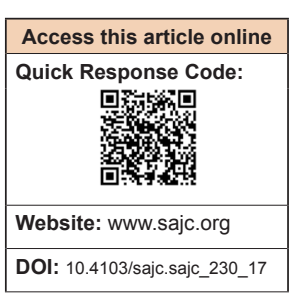

Departments of Medical Oncology, ${ }^{2}$ Radiology and ${ }^{3}$ Laboratory Oncology, Dr. B. R.A. Institute Rotary Cancer Hospital, All India Institute of Medical Sciences, 'Department of Pathology, All India Institute of Medical Sciences, New Delhi, India

Correspondence to: Dr.Ajay Gogia,

E-mail: ajaygogia@gmail.com cytarabine (CODOX-M/IVAC) regimen with or without rituximab (cyclophosphamide, adriamycin, vincristine, high-dose methotrexate/Ifosfamide, cytarabine along with intrathecal therapy), four patients received other chemotherapy protocols and were excluded. We have included patients who received CODOX-M/IVAC (with or without Rituximab) for analysis in this study [Table 1].

\section{Diagnostic and staging procedures}

The demographic details, clinical history, physical examination, and assessment of site of involvement and staging at presentation were recorded. The complete blood cell count, lactate dehydrogenase, electrolytes, liver, and kidney function were noted at presentation. The staging workup included computed tomography scan or positron emission tomography of the whole body, bone marrow aspirate, and biopsy and cerebrospinal fluid (CSF) analysis. Clinical stage was evaluated in accordance with conventional Ann Arbor criteria. The extranodal disease was defined as a contiguous involvement of a nonlymphoid organ. Primary extranodal lymphoma was diagnosed when the initial or major site of disease was in an extranodal organ, and there was none or only regional lymph node involvement. Extensive involvement of any nonlymphoid organ system was taken as Stage IV disease, any lymph nodal mass $>7.5 \mathrm{~cm}$ in longest diameter or a mediastinal mass occupying more than one-third of the thoracic diameter was taken as bulky disease. Toxicity of chemotherapy was assessed as per common toxicity criteria version 4.0.

\section{Clinical response evaluation}

Clinical response was classified as complete remission (CR), partial remission (PR), stable disease (SD), and progressive

This is an open access journal, and articles are distributed under the terms of the Creative Commons Attribution-NonCommercial-ShareAlike 4.0 License, which allows others to remix, tweak, and build upon the work non-commercially, as long as appropriate credit is given and the new creations are licensed under the identical terms.

For reprints contact: reprints@medknow.com

How to cite this article: Patekar M, Gogia A, Tiwari A, Kumar L, Sharma A, Mallick SR, et al. Adult Burkitt lymphoma: An institutional experience with a uniform chemotherapy protocol. South Asian J Cancer 2018;7: 195-9. 
Table 1: Rituximab-cyclophosphamide, vincristine, doxorubicin, methotrexate, ifosfamide, etoposide, cytarabine regimen

\begin{tabular}{|c|c|c|}
\hline Drug & Drug & Days \\
\hline \multicolumn{3}{|c|}{ R-CODOX-M regimen* } \\
\hline $\mathrm{R}$ & $375 \mathrm{mg} / \mathrm{m}^{2} \mathrm{IV}$ & D1 \\
\hline Cyclophosphamide & $800 \mathrm{mg} / \mathrm{m}^{2} \mathrm{IV}$ & D1 \\
\hline Cyclophosphamide & $200 \mathrm{mg} / \mathrm{m}^{2} \mathrm{IV}$ & D2 to $\mathrm{d} 4$ \\
\hline Vincristine & $1.5 \mathrm{mg} / \mathrm{m}^{2}$ (maximum $2 \mathrm{mg}$ ) IV bolus & D1 and $d 8$ \\
\hline Doxorubicin & $40 \mathrm{mg} / \mathrm{m}^{2} \mathrm{IV}$ & D1 \\
\hline Methotrexate & $\begin{array}{l}\text { Age }<65 \text { years: } 3 \mathrm{gm} / \mathrm{m} 3 \text { with } \\
\text { leucovorin rescue } \\
\text { Age }>65 \text { year: } 1 \mathrm{gm} / \mathrm{m} 2 \text { with } \\
\text { leucovorin rescue }\end{array}$ & D10 \\
\hline Intrathecal medications & IT cytarabine $70 \mathrm{mg}$ & D1 and D3 \\
\hline & IT methotrexate $12 \mathrm{mg}$ & D15 \\
\hline \multicolumn{3}{|c|}{ R-IVAC regimen* } \\
\hline $\mathrm{R}$ & $375 \mathrm{mg} / \mathrm{m}^{2}$ & D1 \\
\hline Ifosfamide & $\begin{array}{l}\text { Age }<65 \text { years: } 1500 \mathrm{mg} / \mathrm{m} 2 \mathrm{IV} \\
\text { with mesna } \\
\text { Age }>65 \text { years: } 1000 \mathrm{mg} / \mathrm{m} 2 \mathrm{IV} \text { with } \\
\text { mesna }\end{array}$ & D1- D5 \\
\hline Etoposide & $60 \mathrm{mg} / \mathrm{m}^{2}$ & D1-D5 \\
\hline Cytarabine & $\begin{array}{l}\text { Age }<65 \text { years: } 2 \mathrm{~g} / \mathrm{m} 2 \text { IV every } 12 \\
\text { hours } \\
\text { Age }>65 \text { years: } 1 \mathrm{~g} / \mathrm{m} 2 \text { IV every } 12 \\
\text { hours }\end{array}$ & D1, D2 \\
\hline Intrathecal medications & IT methotrexate $12 \mathrm{mg}$ & D5 \\
\hline
\end{tabular}

disease based on Modified Cheson lymphoma response evaluation criteria. The overall response rate (ORR) was calculated including CR and PR. ${ }^{[2]}$

The time period from the beginning of the treatment to the date of death from any cause or to the date of the last follow-up was defined as overall survival (OS). Progression-free survival (PFS) was defined as the time from the beginning of treatment to disease progression or relapse.

\section{Statistical analysis}

Statistical analysis was done using IBM SPSS version 20. The Chi-square test was used to analyze the correlation between treatment regimen and achievement of response to therapy. Survival was calculated by Kaplan-Meier analysis and factors significantly affecting the survival outcomes were analyzed by Cox's proportional hazard method. Statistical significance was defined as $P<0.05$.

\section{Results}

\section{Patient characteristics}

The median age at presentation was 38 years (19.0-64.0). Elderly patients (Age $>60$ years) constituted $11 \%$ of our study population. The majority of patients were male, with the male to female ratio being 3.5:1. The median duration of symptom duration before the presentation was 2 months (0.2-6 months). Extranodal involvement was seen in all the cases, the bone marrow was most common extranodal (9/18 [50\%]). The other extranodal sites were gastrointestinal (GI) tract (8/18 [44.4\%]), lung (3/18 [16.6\%]), breast, adnexa, liver, central nervous syndrome (CNS), nasopharynx, tonsillar fossa, and bone. Pleural effusion and ascites were present in $5(27.8 \%)$ cases, respectively. The CSF cytology was negative in all cases. In our study population, 4 (22.2\%) patients had Burkitt leukemia whereas one patient had primary GI Lymphoma (ileocecal region).
Majority of our patients (14/18 [77.8\%]) presented with advanced stage (Stage III/IV) of disease. B-symptoms (fever, night sweat, and loss of weight or appetite) were present in $12 / 18(66.6 \%)$ cases, and bulky disease was present in $9 / 18(50 \%)$ of patients. $17 / 18(94.5 \%)$ cases were of high-risk BL. In our study population, only one patient was HIV positive. Most of our patients had poor performance status, 10/18 (55.5\%) patients had Eastern Cooperative Oncology Group performance status of $\geq 2$. Low albumin (serum albumin $<3.5 \mathrm{~g} / \mathrm{dl}$ ) was seen in $6 / 18(33.3 \%)$ cases [Table 2].

Information on fluorescence in situ Hybridization (FISH) for c-MYC (8q24) gene was available in 5/18 (27.7\%) cases; of which $2 / 5(40 \%)$ cases were $t(8,14)$ positive while $2 / 5(40 \%)$ cases did not reveal MYC gene rearrangement, one of these patients was tested only for $t(8,14)$ and turned out to be negative.

Four patients underwent an initial surgery and diagnosis of BL was confirmed on histopathological examination of the surgically resected specimen. Two patients presented with acute intestinal obstruction and underwent exploratory laparotomy, biopsy, and diversion colostomy. One of the patients presented with ileocecal mass and underwent right hemicolectomy. The $4^{\text {th }}$ patient presented with bilateral adnexal mass and omental deposits; she underwent exploratory laparotomy with total abdominal hysterectomy with bilateral salpingo-oophorectomy along with right hemicolectomy.

\section{Treatment and outcome}

Four patients were given prephase chemotherapy with cyclophosphamide and dexamethasone. Two patients initially received one cycle of CHOP (cyclophosphamide, doxorubicin, vincristine and prednisolone), each in view of the delay in the confirmed diagnosis of BL. All the 18 patients included in this study were treated with a uniform protocol using CODOX-M/ South Asian Journal of Cancer • Volume 7 Issue 3 •uly-September 2018 
Table 2: Baseline clinical characteristics

\begin{tabular}{lc}
\hline Variable $(\boldsymbol{n}=\mathbf{1 8})$ & Frequency $(\mathbf{\%})$ \\
\hline Median age, years (range) & $38(19-64)$ \\
Sex & \\
Male & $14(77.8)$ \\
Female & $4(22.2)$ \\
Performance status & \\
$0-1$ & $8(44.4)$ \\
2 or more & $10(55.6)$ \\
Stage & \\
I + II & $4(22.2)$ \\
III + IV & $14(77.8)$ \\
Risk stratification & \\
High risk & $17(94.4)$ \\
Low risk & $1(5.6)$ \\
LDH (IU/L) & \\
Normal & $3(16.7)$ \\
Elevated & $15(83.3)$ \\
Serum albumin $(\mathrm{g} / \mathrm{dl})$ & \\
$>3.5$ & $12(66.6)$ \\
$\leq 3.5$ & $6(33.4)$ \\
BM status & \\
Positive & $9(50.0)$ \\
Negative & $9(50.0)$ \\
CSF status & \\
Positive & 0 \\
Negative & $18(100.0)$ \\
\hline LDH=Lactate dehydrogenase, CSF=Cerebrospinal fluid, BM=Bone marrow
\end{tabular}

$\mathrm{LDH}=$ Lactate dehydrogenase, $\mathrm{CSF}=$ Cerebrospinal fluid, $\mathrm{BM}=$ Bone marrow

IVAC, of these $12(66.6 \%)$ patients received rituximab. The ORR and complete response (CR) rate was $77.8 \%$ and $66.7 \%$, respectively. In the subgroup of patients who received Rituximab RCODOX-M/IVAC (RCODOX-M/IVAC), the ORR, and CR rate was $75.0 \%$, and $66.6 \%$ respectively while the ORR and CR rate in the CODOX-M/IVAC subgroup was 83.3.0\% and $66.6 \%$, respectively. Five (27\%) patients had a progression of disease while on therapy, of these two patients developed new-onset cranial nerve palsy and on evaluation was found to have CNS disease along with nodal and medullary involvement. Of the other three patients, one patient developed new skin lesions and the other developed fulminant hepatic failure, both these patients were on evaluation confirmed to have disease progression. One patient with Burkitt leukemia had persistent bone marrow involvement despite therapy. All these five patients succumbed to their illness.

One patient had a partial response after completion of treatment; he received radiotherapy (36 Gy/20\#) to residual disease. He achieved a CR after completion of radiotherapy.

Out of 18 patients on CODOX-M/IVAC, 14 high-risk patients and one low-risk patient received all 4 cycles and 3 cycles, respectively, i.e., $83.3 \%$ patients were able to complete scheduled treatment. Three high-risk patients received only 3 cycles as they had disease progression.

Toxicity

In our study, $16(88.8 \%)$ patients had grade $3 / 4$ neutropenia, $14(77.7 \%)$ had grade 3/4 thrombocytopenia, $12(66.6 \%)$ patients had febrile neutropenia, and $12(66.6 \%)$ patients had grade $3 / 4$ anemia. Of the nonhematologic toxicity, mucositis was the most significant toxicity, 6 (33.3\%) patients developed grade $3 / 4$ mucositis. The other nonhematologic grade $3 / 4$ toxicity was diarrhea $(15 \%)$ and transaminitis $(5 \%)$.

South Asian Journal of Cancer Volume 7 • Issue 3 • July-September 2018

\section{Survival}

The median event-free survival (EFS) and OS were not reached in our cohort. One-year EFS and OS was $76 \%$ and $81.1 \%$, respectively, with a median follow-up of 15.6 months (2.5-49.2 months) [Figures 1 and 2]. The estimate 2-year EFS and OS are $68.4 \%$ and $73 \%$, respectively. Bone marrow involvement $(P=0.01)$ and female gender $(P=0.01)$ was associated with poor OS.

\section{Discussion}

This is the first case series describing the treatment outcome of the patients of BL using a uniform protocol from India. In our series, the median age of patients was 38 (19-64), while $2 / 18(11 \%)$ patients were elderly (age $>60$ years). The patients were predominantly male (3.5:1). These findings are consistent with other case series. ${ }^{[3-5]}$ In some series, a higher median age (44-58 year) has also been reported. ${ }^{[6,7]}$ Two earlier studies from India have reported the median age of presentation to be 22 years and 6.5 years with BL comprising $3.5 \%$ and $3 \%$ of all NHL, respectively ${ }^{[8,9]}$ However, in these two studies, pediatric BL cases were also included in this study.

In the present study, the patients have a higher frequency of B-symptoms (66.6\%), a higher proportion of advanced disease $(77.8 \%)$, bone marrow involvement $(50 \%)$, and Burkitt leukemia (22\%) subtype. CNS involvement was not seen in our case series. The majority of the patients in our study were high risk $(94.5 \%)$ and bone marrow being the most common extranodal site $(50 \%)$ followed by GI tract $(44.4 \%)$. Studies have reported bone marrow and central nervous system (CNS) involvement in $30 \%-38 \%$ and $13 \%-17 \%$ of adults, respectively. ${ }^{[3,10,11]}$

In our study, all 18 patients received CODOX-M/IVAC regimen out of them, 12 received rituximab. The ORR and CR rate in our population was $75 \%$ and $66.6 \%$, respectively. The 1 -year EFS and OS was $76 \%$ and $81.1 \%$ respectively with a median follow-up of $15.6(2.5-49.2)$ months. It is interesting that all patients who achieved $\mathrm{CR}$ at the end of treatment are disease free and surviving till the last follow-up. This supports the fact that though BL is an aggressive disease, once patients successfully complete treatment the long-term outcomes are good. The results of our study are comparable to the outcome of other studies using this protocol ${ }^{[3,4,12,13,17]}$ [Table 3]. All of these trials used a risk-adapted approach. Two-year EFS and OS varied from 64\%$92 \%$ and $67 \%-73 \%$, respectively in these studies. The Dosing of CODOX-M/IVAC has varied slightly between these studies. Other regimens such as Cancer and leukemia Group B, HyperCVAD (hyperfractionated cyclophosphamide, vincristine, doxorubicin, dexamethasone), Rituxmab-HyperCVAD, Lymphomes Malins B (LMB) reported 2-year EFS and OS varied from $61 \%$ to $74 \%$ and $49 \%$ to $78 \%$, respectively ${ }^{[6,7,14]}$ Dunleavy et al. reported a freedom from progression of $95 \%$ and OS of $100 \%$ at a median follow-up 86 months with DA-EPOCH regimen with rituximab. ${ }^{[15]}$ The impact of rituximab has not been well studied in BL. Few studies reported 3-year EFS and OS in the rituximab-containing arms at $74 \%-76 \%$ and $77 \%-82 \%{ }^{[16-18]}$ [Table 4].

Bone marrow involvement $(P=0.01)$ and female gender $(P=0.01)$ were significantly related to poor OS. The association of poor OS with female gender could be a chance association in view of a small sample size and relatively shorter follow-up. The grade 3/4 myelosuppression was universal CODOX-M/IVAC regimen, and 
Table 3: Regimens and outcomes for the upfront therapy of Burkitt lymphoma

\begin{tabular}{|c|c|c|c|c|c|c|c|}
\hline Reference & Regimen & $n$ & Median age (years) & Risk & TRM & EFS/PFS & OS \\
\hline Mead et al. ${ }^{[3]}$ & CODOX-M/IVAC & 52 & 35 & High risk $77 \%$ & 5 & 2 years EFS $65 \%$ & 2 years OS $67 \%$ \\
\hline Mead et al. ${ }^{[4]}$ & CODOX-M/IVAC & 53 & 37 & High risk $79 \%$ & 9 & 2 years EFS $64 \%$ & 2 years OS $67 \%$ \\
\hline Magrath et al..$^{[12]}$ & CODOX-M/IVAC & 41 & 25 & High risk $83 \%$ & 0 & 2 years PFS 92\% & NA \\
\hline Lacasce et al. ${ }^{[13}$ & CODOX-M/IVAC & 14 & 47 & High risk $78 \%$ & 0 & 2 years EFS $64 \%$ & 2 years OS $71 \%$ \\
\hline Barnes et al. ${ }^{[17]}$ & $\begin{array}{l}\text { CODOX-M/IVAC } \\
\text { With R } \\
\text { Without R }\end{array}$ & $\begin{array}{l}40 \\
40\end{array}$ & 46 & High risk $84 \%$ & NA & $\begin{array}{l}3 \text { years PFS } \\
74 \% \\
61 \%\end{array}$ & $\begin{array}{l}3 \text { years OS } \\
77 \% \\
66 \%\end{array}$ \\
\hline
\end{tabular}

EFS=Event free survival, PFS=Progression free survival, OS=Overall survival, CODOX-M/IVAC=Cyclophosphamide, vincristine, doxorubicin, methotrexate, ifosfamide, etoposide, cytarabine, $\mathrm{NA}=$ Not available, $\mathrm{TRM}=$ treatment related mortality, $\mathrm{R}=$ rituximab

Table 4: Other regimens and outcomes for the upfront therapy of Burkitt lymphoma

\begin{tabular}{|c|c|c|c|c|c|c|c|}
\hline Author & Regimen & $n$ & Median age (years) & Risk & TRM & EFS/PFS & OS \\
\hline Rizzieri et al. ${ }^{[6]}$ & CALGB regimen & 105 & 44 & IPI $\geq 3,47 \%$ & 7 & 2-year EFS 74\% & 3 -year OS 58\% \\
\hline Thomas et al. ${ }^{[7]}$ & Hyper CVAD & 26 & 58 & High LDH 70\% & 5 & 3-year CCR 61\% & 3 -year OS $49 \%$ \\
\hline Diviné et al..$^{[14]}$ & LMB regimen & 72 & 33 & High LDH $60 \%$ & 0 & 2-year EFS 65\% & 2-year OS 70\% \\
\hline Dunleavy et al..$^{[15]}$ & DA-EPOCH & 19 & 25 & High LDH $37 \%$ & 0 & EFS $95 \% * *$ & OS $100 \% * *$ \\
\hline Ribrag et al. ${ }^{[16]}$ & $\begin{array}{l}\text { LMBA regimen } \\
\text { With } \mathrm{R} \\
\text { Without } \mathrm{R}\end{array}$ & $\begin{array}{l}128 \\
129\end{array}$ & 47 & High LDH $75 \%$ & $\begin{array}{l}9 \\
7\end{array}$ & $\begin{array}{l}\text { 3-year EFS } \\
75 \% \\
62 \%\end{array}$ & $\begin{array}{l}\text { 3-year OS } \\
83 \% \\
70 \%\end{array}$ \\
\hline Thomas et al..$^{[18]}$ & R-hyper CVAD & 31 & 46 & High LDH $100 \%$ & 1 & 3-year EFS $80 \%$ & 3 years OS $89 \%$ \\
\hline
\end{tabular}

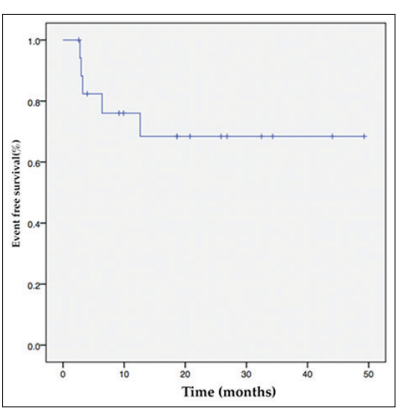

Figure 1: Event-free survival in Burkitt lymphoma

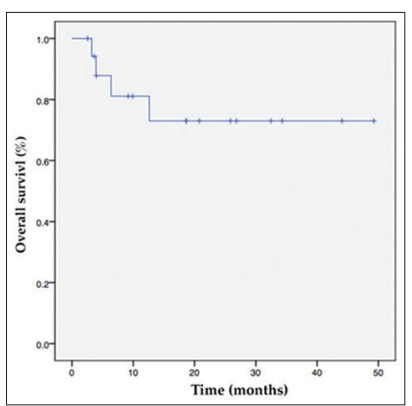

Figure 2: Overall survival in Burkitt Lymphoma it was the most common toxicity in our series. However, despite significant toxicity related to this regimen, the protocol completion rate in our series was $83.3 \%$, which is comparable with western literature. ${ }^{[12,18]}$ There were no toxic deaths in this case series.

A large proportion of our patients were diagnosed of having BL based on morphology and immunohistochemistry. The molecular evidence through FISH was available in only five (27\%) patients. In our resource-limited setting, the availability of FISH for MYC rearrangement hampers adequate molecular diagnosis of these patients.

Our study is limited by its small sample size, short follow-up, and retrospective study design. Despite, of these limitations, it gives us a window of opportunity to see the outcomes and toxicity of our patients who are treated with aggressive chemotherapy protocol like CODOX-M/IVAC.

\section{Conclusion}

Our patients of BL treated with CODOX-M/IVAC protocol are able to complete treatment despite significant toxicity. The outcomes of these patients are comparable to those in the western population. The bone marrow involvement at baseline was found to be a poor outcome predictor in our series which needs verification in larger series.

\section{Financial support and sponsorship}

Nil.

\section{Conflicts of interest}

There are no conflicts of interest.

\section{References}

1. Swerdlow SH, Campo E, Pileri SA, Harris NL, Stein H, Siebert R, et al. The 2016 revision of the World Health Organization classification of lymphoid neoplasms. Blood 2016;127:2375-90.

2. Cheson BD, Pfistner B, Juweid ME, Gascoyne RD, Specht L, Horning SJ, et al. Revised response criteria for malignant lymphoma. J Clin Oncol 2007;25:579-86.

3. Mead GM, Sydes MR, Walewski J, Grigg A, Hatton CS, Pescosta N, et al. An international evaluation of CODOX-M and CODOX-M alternating with IVAC in adult Burkitt's lymphoma: Results of United Kingdom lymphoma group LY06 study. Ann Oncol 2002; 13:1264-74.

4. Mead GM, Barrans SL, Qian W, Walewski J, Radford JA, Wolf M, et al. A prospective clinicopathologic study of dose-modified CODOX-M/ IVAC in patients with sporadic Burkitt lymphoma defined using cytogenetic and immunophenotypic criteria (MRC/NCRI LY10 trial). Blood 2008;112:2248-60.

5. Boerma EG, van Imhoff GW, Appel IM, Veeger NJ, Kluin PM, Kluin-Nelemans JC, et al. Gender and age-related differences in Burkitt lymphoma - Epidemiological and clinical data from the Netherlands. Eur J Cancer 2004;40:2781-7.

6. Rizzieri DA, Johnson JL, Byrd JC, Lozanski G, Blum KA, Powell BL, et al. Improved efficacy using rituximab and brief duration, high intensity chemotherapy with filgrastim support for Burkitt or aggressive lymphomas: Cancer and leukemia group B study 10002. Br J Haematol 2014; 165:102-11.

7. Thomas DA, Cortes J, O'Brien S, Pierce S, Faderl S, Albitar M, et al. Hyper-CVAD program in Burkitt's-type adult acute lymphoblastic leukemia. J Clin Oncol 1999; 17:2461-70.

8. Arora N, Manipadam MT, Nair S. Frequency and distribution of lymphoma types in a tertiary care hospital in South India: Analysis of 5115 cases using the World Health Organization 2008 classification and comparison with world literature. Leuk Lymphoma 2013;54:1004-11.

9. Sahni CS, Desai SB. Distribution and clinicopathologic characteristics of non-Hodgkin's lymphoma in India: A study of 935 cases using WHO classification of lymphoid neoplasms (2000). Leuk Lymphoma 2007;48: 122-33.

10. Blum KA, Lozanski G, Byrd JC. Adult Burkitt leukemia and lymphoma. Blood 2004; 104:3009-20.

11. McMaster ML, Greer JP, Greco FA, Johnson DH, Wolff SN, Hainsworth JD, South Asian Journal of Cancer Volume 7 Issue 3 July-September 2018 
et al. Effective treatment of small-noncleaved-cell lymphoma with high-intensity, brief-duration chemotherapy. J Clin Oncol 1991;9:941-6.

12. Magrath I, Adde M, Shad A, Venzon D, Seibel N, Gootenberg J, et al. Adults and children with small non-cleaved-cell lymphoma have a similar excellent outcome when treated with the same chemotherapy regimen. J Clin Oncol 1996;14:925-34.

13. Lacasce A, Howard O, Lib S, Fisher D, Weng A, Neuberg D, et al. Modified magrath regimens for adults with Burkitt and Burkitt-like lymphomas: Preserved efficacy with decreased toxicity. Leuk Lymphoma 2004;45:761-7.

14. Diviné M, Casassus P, Koscielny S, Bosq J, Sebban C, Le Maignan C, et al. Burkitt lymphoma in adults: A prospective study of 72 patients treated with an adapted pediatric LMB protocol. Ann Oncol 2005;16:1928-35.

15. Dunleavy K, Pittaluga S, Shovlin M, Steinberg SM, Cole D, Grant C, et al.
Low-intensity therapy in adults with Burkitt's lymphoma. N Engl J Med 2013;369: 1915-25.

16. Ribrag V, Koscielny S, Bouabdallah K, Salles G, Casasnovas O, Fornecker LM, et al. Rituximab and dose-dense chemotherapy for adults with Burkitt's lymphoma: a randomised, controlled, open-label, phase 3 trial. Lancet 2016;387:2402-11.

17. Barnes JA, Lacasce AS, Feng Y, Toomey CE, Neuberg D, Michaelson JS, et al. Evaluation of the addition of rituximab to CODOX-M/IVAC for Burkitt's lymphoma: A retrospective analysis. Ann Oncol 2011;22: 1859-64.

18. Thomas DA, Faderl S, O'Brien S, Bueso-Ramos C, Cortes J, Garcia-Manero G, et al. Chemoimmunotherapy with hyper-CVAD plus rituximab for the treatment of adult Burkitt and Burkitt-type lymphoma or acute lymphoblastic leukemia. Cancer 2006; 106: 1569-80. 